To cite this article:

Meers, K., Dejonckheere, E., Kalokerinos, E. K., Rummens, K., \& Kuppens, P. (in press). mobileQ: A free user-friendly application for collecting experience sampling data. Behavior Research Methods. https://doi.org/10.3758/s13428-019-01330-1 


\title{
mobileQ: A Free User-Friendly Application for Collecting Experience Sampling Data
}

\author{
Kristof Meers ${ }^{1}$ \\ Egon Dejonckheere ${ }^{1}$ \\ Elise K. Kalokerinos ${ }^{2}$ \\ Koen Rummens ${ }^{3}$ \\ Peter Kuppens ${ }^{1}$ \\ ${ }^{1}$ KU Leuven, Faculty of Psychology and Educational Sciences \\ 2 The University of Melbourne, Melbourne School of Psychological Sciences \\ ${ }^{3}$ University of Bern, Institute for Psychology
}

Word count $=3619$

Author note

The research leading to the results reported in this paper was supported in part by the Research Fund of KU Leuven (C14/19/054). Elise Kalokerinos is supported by an Australian Research Council Discovery Early Career Researcher Award (DE180100352)

Corresponding author: Kristof Meers, Faculty of Psychology and Educational Sciences, KU Leuven, Tiensestraat 102, Leuven, 3000, Belgium. E-mail: kristof.meers@ kuleuven.be 


\begin{abstract}
In this paper, we introduce mobile $Q$, which is a free, open-source software that our lab has developed to use in experience sampling studies. Experience sampling studies have several strengths and are becoming more widely conducted, but there are few free software options. To address this gap, mobileQ has freely available servers, a web interface, and an Android app. To reduce the barrier to entry, it requires no high-level programming, and uses an easy point-andclick interface. It is designed to be used on dedicated research phones, allowing for experimenter control and eliminating selection bias. In this article, we introduce setting up a study in mobileQ, outline the set of help resources available for new users, and highlight the success with which mobileQ has been used in our lab.
\end{abstract}

Keywords: Experience sampling; smartphones; open-source 
An experimental investigation of needs, of action, or emotions cannot be carried out without taking into account the characteristics of the person, his momentary state, and his psychological environment.

- Kurt Lewin, Principles of Topological Psychology, 1936

In 1936, Kurt Lewin challenged psychology researchers to take a more holistic perspective, moving beyond lab research to understand how people function in real environments. Today, accessible mobile technologies such as smartphones allow experience sampling methods to meet Lewin's challenge. These methods allow insight into participants' momentary states and the contexts in which these states occur, letting researchers to "capture life as it is lived" (Bolger, Davis, \& Rafaeli, 2003). In this paper, we hope to facilitate the increasing uptake of experience sampling methods by introducing mobileQ, a free and user-friendly Android platform for experience sampling.

\section{What is the Experience Sampling Method?}

The experience sampling method (ESM; Csikszentmihalyi \& Larson, 1987) involves sampling participants' experiences in natural environments, in real-time (or close to it), and on multiple measurement occasions (Conner, Tennen, Fleeson, \& Barrett, 2009). ESM is a type of ecological momentary assessment (Shiffman, Stone, \& Hufford, 2008; Stone \& Shiffman, 1994), and falls into a broader group of methods called ambulatory assessment (Trull \& Ebner-Priemer, 2013). As mobile technology becomes more widely available, ESM research is becoming increasingly common: A Web of Science topic search (using the keywords experience sampling, ecological momentary assessment, and ambulatory assessment) netted 4496 articles, 687 of which were from 2018. 


\section{Why Use Experience Sampling?}

Experience sampling addresses two problems typically observed in traditional lab research. First, ESM research increases ecological validity. In studying people as they go about their normal lives, researchers get insight into their "natural habitats", and thus are able to understand factors that cannot easily be reconstructed with artificial manipulations or retrospective questionnaires (Czikszentmihalyi \& Larson, 2014; Trull \& Ebner-Priemer, 2013). In addition, real-life contexts are more diverse and less constrained than lab studies, allowing researchers to observe a wider range of responding (Reis \& Gosling, 2010). Second, because participants respond in (or close to) the moment, ESM research reduces recall bias. Many studies have demonstrated that the accuracy of the momentary reports generated for ESM is higher than traditional retrospective methods (e.g. Solhan, Trull, Jahng, \& Wood, 2009; Stone et al., 1998). In addition, ESM research allows researchers to answer different types of research questions. Using ESM, researchers can model the time course of constructs, and explore dynamic phenomena (e.g., how does positive affect change throughout the day?; Trull \& Ebner-Priemer, 2013). ESM can also be used to better understand within-person processes, and to separate these processes from between-person processes (e.g., how do various emotion regulation strategies impact negative affect, and does this differ between depressed and healthy individuals?; Conner et al., 2009). Within-person assessment allows a better understanding of the role of changing contexts, as well as increasing statistical power. Event-based ESM designs also lets researchers capture certain low frequency behaviors and events (e.g., how do partners emotionally cope after a marital conflict?; Bolger \& Laurenceau, 2013), providing richer descriptive data and a deeper understanding of real environments. Finally, ESM research can be used to aggregate over time, providing an improvement on methods that ask people to retrospect on their behavior over a 
time-span (e.g., how many times did you exercise over the last month?), and thus increasing the accuracy of participants' responses (Bolger et al., 2003).

\section{Programs for Experience Sampling}

Given the empirical strength of these methods, and their increasing popularity, it is important that good ESM research tools are available. In the past, ESM research used paper and pencil booklets in combination with a pager, and more recently, palmtops and text messages.

With the advent of widely available smartphone technology, there are now several smartphone options available for experience sampling, although not all options are tailored for psychology research. Arslan, Tata, \& Walther (2018) provide an overview of the options that are available. However, we find that many of these existing options have some combination of three potential drawbacks. In this paper, we introduce our own program, mobileQ, which addresses each of these drawbacks, and was designed based on the long experience of our group in conducting ESM research.

First, existing programs often require payment to use. Some software charges a one-time fee, and some software charges for going over a certain number of participants or signals. In a less direct cost, some software requires a constant mobile data connection and so incurs costs to participants' data plans. In contrast, mobileQ is completely free and open-source[1]. We provide servers, a web interface, and an Android application. No internet connection is needed: data are stored locally, and surveys are sent based on the local notification system, and so there are no data plan costs. Here, we should note that the recent release of the ESM platforms ExperienceSampler (Thai \& Page-Gould, 2017), formr (Arslan, Tata, \& Walther, 2018), Paco (Evans, 2012), aware (Ferreira, Kostakos, Dey, 2015), ohmage (Hicks, 2011), beiwe (Torous, Kiang, Lorme, Onnela) and SEMA3 (Koval et al, 2019) have provided other good free options 
for ESM researchers (although some of these options require a data plan or internet connection). However, these programs do have at least one of the two other potential drawbacks that we discuss in the following paragraphs (for a feature-based comparison of these programs with mobileQ, see Arslan, Tata, \& Walther, 2018; https://comparison-to.formr.org).

Second, existing software options often require some form of higher-level programming. Thus, these options require a time investment to learn the requisite skills, or some existing expertise. In contrast, mobileQ studies are programmed using a point-and-click web interface. No coding is required, and thus the program can be picked up and used very quickly.

Third, the existing software is designed to run on the participants own compatible smartphones. In contrast, mobileQ is designed to be installed on dedicated research phones owned by the lab. Because it was designed with dedicated research phones in mind, mobileQ works perfectly in "kiosk mode" which allows researchers to replace the home screen of the phone with mobileQ, and to lock participants out of accessing or using any other applications on the phone. This feature increases uniformity across participants and prevents distractions or interference from other applications. It also means that when participants unlock the phone, they only see the application pinned to the screen, making it very clearly a research device. To our knowledge, other open-source software has not been designed with this kind of use in mind. We tend to use mobileQ on dedicated research smartphones. One reason for this is that relying on participants to own phones introduces selection bias. Although smartphone adoption is high among young people in Western countries, requiring people to have a smartphone to participate in research studies can miss some segments of the population (e.g. older people, lower SES groups), as well as being problematic in countries where smartphone adoption is lower. Thus, providing phones avoids selection biases. In our experience running ESM studies, 
we have also seen several other advantages of this approach. Most centrally, this approach provides better control over the study: the survey notifications are very difficult to miss or ignore because we can set up the phone appropriately. In addition, participants cannot remove the app from their phone, fiddle with settings that might inhibit notifications (e.g. battery saving modes), or turn off notifications for the app. In addition, all participants see the items displayed in the same way on the same size screen, something that could be particularly important with scales like sliders, where small screen sizes could reduce the range of responding. Because these dedicated phones are set up to only run mobileQ, the phone battery lasts much longer (over a week with our phones), something that further reduces the chance of missed signals. Finally, dedicated phones mean that our app is not affected by participants updating to the newest version of their phone software, something that can interfere with studies where participants bring their own phones.

We also note that the need to buy dedicated research phones does introduce a cost, and thus is a potential disadvantage of our approach. However, we see this as a minimal disadvantage: mobileQ runs on very basic Android phones (e.g. the Motorola Moto G7 Play, $\$ 130$ USD), and the phones can last a long time: in our group, we are still using phones purchased 8 years ago. We use our phones many times across multiple studies, and so view this as a one-off cost for the lab, although we note that buying phones would not be a cost-effective approach for labs that are seeking only to run a single ESM study. We also run our participants in waves, rather than all at once, so we do not need to have as many phones as we have study participants. This means that our Ns are not restricted by the number of phones that we have in our lab, although it does increase the length of data collection. Overall, we find that phone costs 
are very low when compared with what we pay participants, and so we believe that the phone costs are justified given the advantages of this approach in our situation.

However, we note that there are also some other potential disadvantages of this approach: participants may dislike carrying two phones, they have to remember to carry the phone and charge it (if necessary), and they need to come into the lab to pick up the phone and return it at the end of the study. Researchers should decide whether dedicated lab phones or participant phones best fit their study situation: at this stage, we are unaware of any direct empirical evidence that suggests differences in compliance or satisfaction between the two approaches. If researchers intend to use participant phones, they should be aware that mobileQ is currently Android-only (i.e. it does not work on iOS).

\section{Setting up your own Experience Sampling Study with mobileQ}

In the next section, we will guide you through the most important steps when creating your own study with mobileQ, introducing both the general framework of this software program, as well as covering its basic functionalities. For a detailed, step-by-step tutorial, however, we refer to our set of online instructional videos, which provides an in-depth and up-to-date explanation of all study features (https://mobileQ.org/documentation/instructionalvideos). Additionally, in the web interface, we have implemented intuitive hover-over messages for important key concepts, equipping you with real-time information in the process of setting up a study.

\section{Project Overview}

The mobileQ web interface can be accessed via https://mobileQ.org. After signing in with your personal credentials, the project overview appears, displaying all the ESM research projects you are currently involved in. In this window, the researcher may wish to either modify an 
existing project or to create a new study. When setting up a new study, you are required to provide a project title, a brief description, and a helpdesk e-mail address (i.e. an address that will be displayed on participants' mobile phones that they can contact in case of problems). This study information will later appear when opening the project, and will be complemented with additional study details, such as the current number of enrolled participants, their average compliance rate, and the names of other researchers involved.

\section{Creating a Survey}

Once a project is set up, you can create an ESM survey for participants to complete on their mobile devices. Multiple surveys may be created within the same project. This functionality is particularly convenient when your study consists of different participant conditions (e.g. a unique survey for each condition), your study design requires multiple questionnaires (e.g. a morning versus evening survey), or you wish to combine various types of sampling in your protocol (e.g. combining event-based sampling, where participants initiate a survey upon the occurrence of a specific event such as a social encounter or having had a meal, and timecontingent sampling, where surveys are initiated based on the schedule decided by the researcher). Once a general survey space is set up, you can define its appropriate structure, as well as design the specific items for your questionnaire. Both functionalities rely on an intuitive drag-and-drop system.

Defining the questionnaire structure. First, the structure of your survey needs to be established. In the simplest case, all questions will be located in the main root folder, but you may wish to create various branch folders in which particular questions will be grouped together. The randomization function allows you to present items in a random order, both within and between subfolders. Finally, you can apply a conditional logic to particular questions, with the 
presentation of the next item being dependent on a participant's response to the previous question. In this way, you can set up your own unique flow for your questionnaire.

Designing specific questions. Once the appropriate tree structure is set up, you can provide each folder with the appropriate questions. mobileQ allows you to choose from a wide range of question types relevant for your study. To date, you can provide participants with a simple text message or instruction (info), ask them to choose from a series of suggested alternatives, either selecting a single (single choice) or multiple options (multiple choice), let them rate a question on a continuous scale (slider) or in a two-dimensional grid (grid), or allow them to answer with a written text response (text). Each question can be edited further by applying basic text-formatting, providing appropriate anchors or choice-options (and define the corresponding output values), as well as assigning the question a unique label, which will serve as the variable name in your output dataset.

Versioning. When you create a new survey, the first version is automatically and logically labeled as version 1 . Changes can be made in the same version up to the point where you assign a survey to a trigger template (see next section). Once a questionnaire is appointed to a trigger template, only a newly created version of the survey can be modified. To put the adapted questionnaire into practice, the most recent version needs to be assigned again to the trigger template. In this way, this versioning process prevents you from unintentionally changing the design of a questionnaire that is currently operational, avoiding the potential mix-up of results of different versions of your survey.

\section{Trigger Template}

A trigger template configures the temporal properties of your time-contingent event sampling ESM protocol. Irrespective of a particular start date, it allows you to define the 
moments a questionnaire will be presented for completion. The following time-related settings need to be specified: the day numbers on which a survey needs to be completed (days; e.g. 1,2,3), between which hours you want participants to receive a survey (start / end time; e.g. 9:00AM - 9:00PM) and the amount of triggers in that period (number of beeps; e.g. 10). Day numbers will be converted to dates per participant group (see next section). You will also need to select the appropriate timing type for your survey. The fixed timing type allows you to launch a survey at a specific moment, whereas the random timing type results in a stratified random interval scheme (i.e. the duration between start and end time is divided into a number of equal intervals and one survey is randomly programmed within each interval). Finally, the preferred version of a survey needs to be assigned to the trigger template. Importantly, a project can contain multiple trigger templates, allowing you to present a participant with different surveys during the ESM protocol. Besides deciding on the temporal properties, you also determine here whether you make a participant-initiated demo version of the survey available. This demo option also allows to incorporate event-based sampling in a study.

\section{Participant Group}

With the creation of a participant group, you can set up a group of participants that start the same trigger template on a particular date. Day 1 of the trigger template corresponds to the start date of the participant group. This allows you to enroll different groups of participants on a different start date while assigning them to the same trigger template. When you want to track two (or more) participant subgroups for a different duration, you can couple each subgroup to a separate trigger template. By assigning a unique number to a participant, you can add participants to a participant group. You can override the daily start/end time of the trigger template by specifying a participant's sleep and wake up time separately for weekdays and 
weekends. If you have an experimental design with multiple groups, you will have to manually randomly assign participants to a participant group. You would then create groups on mobileQ based on that manual random assignment: mobileQ currently does not have any features enabling automatic random assignment to groups.

\section{Setting up Mobile Devices}

After adding a participant to a participant group, the participant and his or her mobile device can be paired simply by scanning the QR-code with the mobileQ application. Once synchronized, the mobile device is ready for deployment. If made available in the trigger template, the mobileQ application menu now offers a demo version of the survey. This functionality is very convenient when briefing a participant about the study. Also, participants can choose how they wish to be notified when a survey is available for completion: by sound, vibration, or a combination of both (one of the two must always be on to ensure that the participant is notified).

\section{Results}

The results section provides an overview of the participants enrolled in your ESM study, how many surveys each participant received, and how many of the surveys were completed. The ratio of the last two parameters reflects a participant's compliance, which is available in the result section as a percentage accompanied by a clear visual presentation. Without having to export the data, you can check on a person-level when the surveys are scheduled, which ones are already filled in, and what answers were given. Of course, your data are available for downloading in .csv, .ods, and .xlsx format at any time during the study. If, for whatever reason, a participant quits the study earlier than scheduled, the mobile device can be decoupled by a single mouse click. 


\section{Performance}

mobileQ has been extensively piloted and used by both our own research group and national and international collaborators. This extensive pre-publication use has allowed us to debug the software, examine its performance under highly varying conditions (in terms of mobile phone type, survey type, user language, time-zones, different types of designs, using both community and patient samples, etc.), and optimize user experience.

So far, mobileQ has successfully programmed and processed 198,628 smartphone surveys, amounting to a total of 6,769,260 recorded responses. It has been tested and used in research on a variety of recent Android smartphones, including Motorola, Samsung, Sony, HTC, LG, OnePlus, WileyFox, and Huawei.

mobileQ has been instrumental in research using a wide variety of ESM protocols and designs, including straightforward 1-week ESM protocols (e.g., Koval, et al., 2015), long term multiple wave measurement burst studies (e.g., Dejonckheere et al., 2018; Erbas, et al., 2018), sampling around specific life-events (e.g., Kalokerinos, Erbas, Ceulemans, \& Kuppens, 2019), between-subject manipulations of the ESM protocol (e.g., De Vuyst, et al., 2019), and simultaneous sampling of romantic couples (e.g., Sels, et al., in press). It has also been used to address a variety of research questions in daily life, for example the within-person structure of affect (e.g., Kuppens, et al., 2013), the situated nature of emotion and goals (e.g., De Leersnyder, et al., 2018), the dynamics of emotional experience (e.g., Dejonckheere et al., 2018; Houben \& Kuppens, in press), the impact of mental health intervention (e.g., Van der Gucht, et al., 2019). It has been used with both healthy samples and samples with psychiatric disorder (e.g., Heininga et al., 2019; Dejonckheere, Kalokerinos, Bastian \& Kuppens, 2019; Dejonckheere, et al., 2019). 
We have also piloted mobileQ with researchers with no experience with the platform, and found that they were able to program surveys and load them onto smartphones with minimal instruction. Nevertheless, help resources are available to get researchers started. In addition to the hover-over information and video tutorials mentioned above, the publication of MobileQ comes with a platform that allows the novice user to ask questions to fellow users, and expert users to help novice users (and will be monitored by expert users from our network).

\section{Lifecycle}

mobileQ has been used in our research group since 2011, and we have been using the experience sampling method since 2003. We intend to use mobileQ for our future ESM research for many years to come, and we anticipate adding more features in the future as our research progresses. In this period, we also aim to provide mobileQ as a service for research use. As the added cost for extra studies is very low, we are able to provide the service to researchers for free. In case of particularly large studies, we might ask you to run them on your own infrastructure. More up-to-date information about the lifecycle can be found in the software license agreement on the mobileQ website.

\section{Conclusion}

By making mobileQ freely available to the research community, we hope to help facilitate the adoption of experience sampling research methods to study the nature of human behaviors, thoughts, and feelings in their "natural habitat", daily life. mobileQ offers a versatile and easy-to-use platform that allows the novice researcher to design a wide variety of experience sampling studies and to program Android smartphones to run them without technical background knowledge. At the same time, the availability of open-source code allows more adventurous 
researchers or developers to add new features or customize it further to accommodate their own interests, formats, or devices. 


\section{References}

Arslan, R.C., Tata, C.S. \& Walther, M.P. (2018). formr: A study framework allowing for automated feedback generation and complex longitudinal experience sampling studies using R. doi:10.5281/zenodo.3229668

Bolger, N., Davis, A., \& Rafaeli, E. (2003). Diary methods: Capturing life as it is lived. Annual Review of Psychology, 54, 579-616. doi:10.1146/annurev.psych.54.101601.145030

Bolger, N., \& Laurenceau, J. P. (2013). Intensive Longitudinal Methods. New York, NY: Guilford.

Conner, T. S., Tennen, H., Fleeson, W., \& Barrett, L. F. (2009). Experience sampling methods: A modern idiographic approach to personality research. Social and Personality Psychology Compass, 3, 292-313. doi: 10.1111/j.1751-9004.2009.00170.x

Csikszentmihalyi, M., \& Larson, R. (2014). Validity and reliability of the experience-sampling method. In M. Csikszentmihalyi (Ed.) Flow and the Foundations of Positive Psychology (pp. 35-54). The Netherlands: Springer Netherlands.

Csikszentmihalyi, M., \& Larson, R. (1987). Validity and reliability of the experience-sampling method. Journal of Nervous and Mental Disease, 175, 526-536. http://dx.doi.org/10.1097/00005053-198709000-00004

Dejonckheere, E., Kalokerinos, E. K., Bastian, B., \& Kuppens, P. (2018). Poor emotion regulation ability mediates the link between depressive symptoms and affective bipolarity. Cognition and Emotion, 33, 1076-1083.

https://doi.org/10.1080/02699931.2018.1524747 
Dejonckheere, E., Mestdagh, M., Houben, M., Erbas, Y., Pe, M., Koval, P., Brose, A., Bastian, B., \& Kuppens, P. (2018). The bipolarity of affect and depressive symptoms. Journal of Personality and Social Psychology, 114, 323-341.

Dejonckheere, E., Mestdagh, M., Houben, M., Rutten, I., Sels, L., Kuppens, P., \& Tuerlinckx, F. (2019). Complex affect dynamics add limited information to the prediction of psychological well-being. Nature Human Behaviour, 3, 478-491.

De Leersnyder, J., Koval, P., Kuppens, P., \& Mesquita, B. (2018). Emotions and concerns: Situational evidence for their systematic co-occurrence. Emotion, 18, 597-614.

De Vuyst, H-J., Dejonckheere, E., Van der Gucht, K., \& Kuppens, P. (2019). Does repeatedly reporting positive or negative emotions in daily life have an impact on the level of emotional experiences and depressive symptoms over time? PLoS ONE, 14, e0219121, 112.

Erbas, Y., Ceulemans, E., Kalokerinos, E., Houben, M., Koval, P., Pe, M. L., \& Kuppens, P. (2018). Why I don't always know what I'm feeling: The role of stress in within-person fluctuations in emotion differentiation. Journal of Personality and Social Psychology, $115,179-191$.

Evans, B. (2012). Paco: The personal analytics companion [Computer software]. Retrieved from https://pacoapp.com

Ferreira, D., Kostakos, V., \& Dey, A.K. (2015). AWARE: Mobile context instrumentation framework. Frontiers in ICT, 2(6), 1-9. doi:10.3389/fict.2015.00006

Heininga, V. E., Dejonckheere, E., Houben, M., Obbels, J. Sienaert, P., Leroy, B. Van Roy, J., \& Kuppens, P. (2019). The dynamical signature of anhedonia in Major Depressive 
Disorder: Positive emotion dynamics, reactivity, and recovery. BMC Psychiatry, 19, 211. doi:10.1186/s12888-018-1983-5

Hicks, J., Ramanathan, N., Falaki, H., Longstaff, B., Parameswaran, K., Monibi, M., Kim, D. H., Selsky, J., Jenkins, J., Tangmunarunkit, H., Estrin, D. (2011) Ohmage: An open mobile system for activity and experience sampling. Center for Embedded Networked Sensing, Technical Report \#100.

Houben, M., \& Kuppens, P. (in press). Emotion dynamics and the association with depressive features and borderline personality disorder traits: Unique, specific and prospective relationships. Clinical Psychological Science.

Kalokerinos, E. K., Erbas, Y., Ceulemans, E., \& Kuppens, P. (2019). Differentiate to regulate: Low negative emotion differentiation is associated with ineffective use but not selection of emotion-regulation strategies. Psychological Science, 30, 863-879.

Koval, P., Brose, A., Pe, M. L., Houben, M., Erbas, Y., Champagne, D., \& Kuppens, P. (2015). Emotional inertia and external events: The roles of exposure, reactivity, and recovery. Emotion, 15, 625-636.

Koval, P., Hinton, J., Dozo, N., Gleeson, J., Alvarez, M., Harrison, A., Vu, D., Susanto, R., Jayaputera, G., \& Sinnott, R. (2019). SEMA3: Smartphone Ecological Momentary Assessment, Version 3. [Computer software]. Retrieved from http://www.sema3.com Kuppens, P., Tuerlinckx, F., Russell, J. A., \& Barrett, L. F. (2013). The relation between valence and arousal in subjective experience. Psychological Bulletin, 139, 917-940.

Lewin, K. (1936). Principles of Topological Psychology. United Kingdom: Read Books Ltd. 
Reis, H. T., \& Gosling, S. D. (2010). Social psychological methods outside the laboratory. In S. T. Fiske, D. T. Gilbert, \& G. Lindzey (Eds.) Handbook of Social Psychology (pp. 82114). Hoboken, NJ: John Wiley \& Sons.

Sels, L., Cabrieto, J., Butler, E., Reis, H., Ceulemans, E., \& Kuppens, P. (in press). The occurrence and correlates of emotional interdependence in romantic relationships. Journal of Personality and Social Psychology.

Shiffman, S., Stone, A. A., \& Hufford, M. R. (2008). Ecological momentary assessment. Annual Review of Clinical Psychology, 4, 1-32.

Solhan, M. B., Trull, T. J., Jahng, S., \& Wood, P. K. (2009). Clinical assessment of affective instability: Comparing EMA indices, questionnaire reports, and retrospective recall. Psychological Assessment, 21, 425-436. doi: 10.1037/a0016869

Stone, A. A., Schwartz, J. E., Neale, J. M., Shiffman, S., Marco, C. A., Hickcox, M., ... Cruise, L. J. (1998). A comparison of coping assessed by ecological momentary assessment and retrospective recall. Journal of Personality and Social Psychology, 74, 1670-1680. doi: 10.1037/0022-3514.74.6.1670

Stone, A. A., \& Shiffman, S. (1994). Ecological momentary assessment (EMA) in behavioral medicine. Annals of Behavioral Medicine, 16, 199-202.

Thai, S., \& Page-Gould, E. (2017, June 15). ExperienceSampler: An open-source scaffold for building smartphone apps for experience sampling. Psychological Methods. Advance online publication. doi: 10.1037/met0000151

Torous, J., Kiang, M. V., Lorme, J., \& Onnela, J.-P. (2016) New tools for new research in psychiatry: A scalable and customizable platform to empower data driven smartphone research. JMIR Mental Health, 3(2), e16, 1-17. doi: 10.2196/mental.5165 
Trull, T. J., \& Ebner-Priemer, U. (2013). Ambulatory assessment. Annual Review of Clinical Psychology, 9, 151-176. doi: 10.1146/annurev-clinpsy-050212-185510

Van der Gucht, K., Dejonckheere, E., Erbas, Y., Takano, K., Vandemoortele, M., Maex, E., Raes, F., \& Kuppens, P. (2019). An experience sampling study examining the potential impact of a mindfulness-based intervention on emotion differentiation. Emotion, 19, 123131.

[1] The open-source version of the code will be made available on mobileQ.org in the course of 2019 (pending clean-up, removal of KU Leuven references, and licensing information). Upon request, it can easily be made available to a reviewer of this manuscript. 